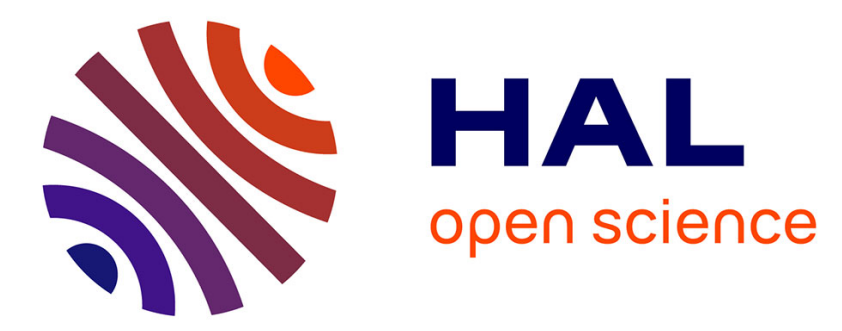

\title{
Applying the concept of uncertainty to the sFlt-1/PlGF cut-offs for diagnosis and prognosis of preeclampsia
}

\author{
Pacifique Lévy, Safouane Hamdi, Jean Guiboudenche, Marie Clothilde
}

Haguet, Sophie Bailleul, Guillaume Lefèvre

\section{- To cite this version:}

Pacifique Lévy, Safouane Hamdi, Jean Guiboudenche, Marie Clothilde Haguet, Sophie Bailleul, et al.. Applying the concept of uncertainty to the sFlt-1/PlGF cut-offs for diagnosis and prognosis of preeclampsia. Clinical Chemistry and Laboratory Medicine, 2021, 59 (4), pp.681-686. 10.1515/cclm2020-0477 . hal-03359360

\section{HAL Id: hal-03359360 \\ https://hal.sorbonne-universite.fr/hal-03359360}

Submitted on 30 Sep 2021

HAL is a multi-disciplinary open access archive for the deposit and dissemination of scientific research documents, whether they are published or not. The documents may come from teaching and research institutions in France or abroad, or from public or private research centers.
L'archive ouverte pluridisciplinaire HAL, est destinée au dépôt et à la diffusion de documents scientifiques de niveau recherche, publiés ou non, émanant des établissements d'enseignement et de recherche français ou étrangers, des laboratoires publics ou privés. 
Applying the concept of uncertainty to the sFIt-1/PIGF cut-offs for diagnosis and prognosis of preeclampsia

Levy Pacifique ${ }^{1}$, Hamdi Safouane ${ }^{3}$, Guibourdenche Jean ${ }^{3}$, Haguet Marie Clotilde ${ }^{4}$, Bailleul Sophie ${ }^{1}$, Lefèvre Guillaume ${ }^{1}$

1. Biochemistry Department, Tenon Hospital, AP-HP, Sorbonne University, Paris, France.

2. Biochemistry Department, CHU Toulouse, Toulouse III University, Toulouse, France.

3. Hormonology Department, Cochin Hospital, AP-HP, University of Paris, Paris, France.

4. Biochemistry Department, Armand Trousseau Hospital, AP-HP, Sorbonne University, Paris, France.

Five pages, 2866 words, three tables, one figure, one supplement, 25 references

Objectives: Placental growth factor (PIGF) and soluble fms-like tyrosine kinase-1 (sFlt-1) assays and the corresponding ratios (sFlt-1/PIGF) have been proposed to aid in the diagnosis by exclusion and/or prognosis of preeclampsia (PE). A method for evaluating ratio uncertainties (RUs), based on the theory of error propagation, was applied to the sFlt-1/PIGF ratio.

Methods: RUs were calculated using data derived from sFIt-1 and PIGF Internal Quality Control (IQC) results collected from four centers using Elecsys (Roche) or Kryptor (Thermo Fisher) sFlt1 and PIGF assays. The corresponding RUs were defined for each ratio value.

Results: the RUs increased linearly with the sFIt-1/PIGF ratio values. The Elecsys RUs were lower than the Kryptor RUs. Although RUs cannot eliminate differences in ratio values observed among various immunoassays, it can affect interpretation of the sFIt-1/PIGF ratio, especially when results are within the range of predefined PE diagnosis or prognosis cut-offs. Since RUs are only a function of PIGF and sFlt-1 precision, they can be calculated for each assay from each laboratory to adjust the interpretation of sFIt-1/PIGF ratio results in the context of PE. 


\section{Introduction}

Preeclampsia (PE) is one of the most common potentially lethal diseases of pregnancy and occurs in $3-5 \%$ of all the pregnancies worldwide [1]. It is characterized by the new onset of hypertension and proteinuria after 20 weeks of gestation [2]. Diagnosing PE remains a challenge because its two main diagnostic criteria, hypertension and proteinuria, are not specific. Several studies have demonstrated the strong association between angiogenic factor imbalance, soluble fms-like tyrosine kinase-1 (sFlt1) and placental growth factor (PIGF) and the onset of this disease [3, 4]. The sFlt-1/PIGF ratio is therefore now proposed as an effective marker to rule out a diagnosis or confirm a prognosis of $\mathrm{PE}$ and is used by some physicians as a component of PE monitoring [5]. Generally, cut-offs are defined in studies where sampling, procedures, assays and protocols are optimized. This is no longer the case in routine practice where reagents, calibration and pre-analytical procedures differ from those encountered in the original studies. For any biological result, including sFIt-1/PIGF and its corresponding ratio, there is an uncertainty associated with each test. In the context of PE, the sFlt1/PIGF ratio has been chosen to optimize the diagnostic values of angiogenic factors, but no study has defined the uncertainty associated with ratio results. One way laboratories can timely evaluate and monitor the dispersion of measurements is to use internal quality controls (IQC), which estimates assay precision at a regular rate [6]. Several measurements could be used to calculate the standard deviation (SD) for a result and, assuming the data are normally distributed, the probability that the difference between the true value and the measured value is greater than two standard deviations is less than 5\% [7]. For a given assay, the laboratory can calculate the SD for several levels to encompass a measurement range that can be encountered in clinical situations.

In the case of a ratio between assays, the uncertainty of the denominator and numerator pair can be different. A slight change in the denominator value (PIGF) can have a huge effect on the sFIt-1/PIGF ratio value, especially when the denominator value is low, a case which is precisely encountered with PIGF in PE. Furthermore, contrary to sFIt-1, bias among PIGF results observed between immunoassays complicates the interpretation of ratio results [8]. As proposed by Shackleford et al., ratio uncertainty between assays, i.e. the ratio variance of the first-order Taylor approximation, can be calculated with two different statistical methods using the so-called "propagation of error" approach [9]. Here, we present the application of this statistical method to determining sFIt-1/PIGF ratio uncertainty. 


\section{Materials and Methods:}

sFIt-1 and PIGF IQC data were collected from four separate university clinical chemistry laboratories: Tenon (Paris, center 1) and Purpan (Toulouse, center 2) hospitals, which use Thermo Fisher Kryptor assays; and Cochin (Paris, center 3 ) and Trousseau (Paris, center 4 ) hospitals, which use Roche Elecsys assays. Roche sFlt-1 and PIGF IQC were determined using an Elecsys analyzer ( $n=2$ IQC levels). Thermo Fisher sFIt-1 and PIGF IQC were determined using a Kryptor compact PLUS analyzer ( $n=3$ IQC levels). Ratio uncertainties (RU) were calculated, by either the variance of the first-order Taylor approximation to the ratio which allows the calculation of corresponding ratio $S D(S D(R))$

$\mathrm{SD}(R)=\sqrt{\frac{\operatorname{Var}(\mathrm{x}) \mu_{y}^{2}}{\mu_{x}^{4}}+\frac{\operatorname{Var}(\mathrm{y})}{\mu_{x}^{2}}}$

or the mathematical equivalent of the "propagation of error" (POE) [9]

$\mathrm{POE}=\left(\frac{\mu_{y}}{\mu_{x}}\right) \sqrt{\left(\frac{\mathrm{SD}(\mathrm{x})}{\mu_{x}}\right)^{2}+\left(\frac{\mathrm{SD}(\mathrm{y})}{\mu_{y}}\right)^{2}}$

Nine possible sFlt-1/PIGF ratio associations were calculated from the sFlt-1 and PIGF IQC results. They corresponded to a low PIGF level associated with high, intermediate and low sFlt-1 levels; an intermediate PIGF level associated with high, intermediate and low sFIt-1 levels; and a high PIGF level associated with high, intermediate and low sFlt-1 levels. The sFIt-1/PIGF SD (R) and POE were calculated for each association. sFIt-1/PIGF RU was defined as the ratio value $\pm 2 \mathrm{SD}(\mathrm{R})$ corresponding to a range where the estimate of the true ratio value was found with a probability of $95 \%(95 \% \mathrm{Cl})$.

A Thermo Fisher Kryptor platform was used for centers 1 and 2, and a Roche Cobas platform was used for centers 3 and 4 . The Thermo-Fisher sFIt1 and PIGF assays were conducted for 9 minutes and 29 minutes, respectively. Both Roche Elecsys assays were conducted for 18 minutes. The sFIt-1 and PIGF measuring ranges for Thermo Fisher Kryptor were 10 to $85,000 \mathrm{ng} / \mathrm{l}$ and 22 to $90,000 \mathrm{ng} / \mathrm{l}$, respectively. For Roche Elecsys, they were 3 to $10,000 \mathrm{ng} / \mathrm{l}$ and 3.6 to $7000 \mathrm{ng} / \mathrm{l}$, respectively. The sFIt-1 and PIGF limits of detection for Thermo Fisher Kryptor were $10 \mathrm{ng} / \mathrm{l}$ and $20 \mathrm{ng} / \mathrm{l}$, respectively. For Roche Elecsys, they were $3 \mathrm{ng} / \mathrm{l}$ and $3.6 \mathrm{ng} / \mathrm{l}$, respectively. sFlt-1 center 1 (Thermo-Fisher Kryptor IQC) gave a CV (\%) of 5.00 to $5.37 \%$ (concentration range: 1474 to $9612 \mathrm{ng} / \mathrm{l}$ ) and center 2 (ThermoFisher Kryptor IQC) gave a CV (\%) of 3.0 to $4.2 \%$ (concentration range: 1159 to $53642 \mathrm{ng} / \mathrm{l}$ ). sFlt-1 center 3 (Roche Elecsys IQC) gave a CV (\%) of 1.64 to $4.21 \%$ (concentration range: 108 to $998 \mathrm{ng} / \mathrm{l}$ ) and center 4 (Roche Elecsys) gave a CV (\%) of 1.79 to 2.84\% (concentration range: 101 to $1011 \mathrm{ng} / \mathrm{l}$ ). PIGF center 1 (Thermo-Fisher Kryptor IQC) gave a CV (\%) of 4.72 to $6.98 \%$ (concentration range: 32.37 to $423.46 \mathrm{ng} / \mathrm{l}$ ) and center 2 gave a CV (\%) of 1.8 to $5.7 \%$ (concentration range: 32 to $413 \mathrm{ng} / \mathrm{l}$ ). PIGF 
center 3 (Roche Elecsys IQC) gave a CV (\%) of 1.41 to $3.91 \%$ (concentration range: 102 to $925 \mathrm{ng} / \mathrm{l} \mathrm{ng} / \mathrm{l}$ ) and center 4 gave a CV (\%) of 3.03 to $3.51 \%$ (concentration range: 105 to $972 \mathrm{ng} / \mathrm{l}$ ).

\section{Results:}

A total of five Kryptor IQC batches ( $n=3$ levels) and four Roche IQC batches ( $n=2$ levels) for sFIt-1 and PIGF were collected (Table 1). Forty-five different sFIt-1/PIGF ratios were calculated with Kryptor and 16 with Elecsys IQC results. The SD(R) and POE results were calculated for each possible ratio. All the IQC results and corresponding SDs are given in the supplement to Table 1. As demonstrated by Shackleford et al., SD(R) and POE gave the same values. The relationship between the ratio uncertainty and the sFIt-1/PIGF ratio for both Kryptor and Roche is illustrated in Figure 1. For the Kryptor assay, the ratio equations corresponding to the upper bound (UB) of the ratio uncertainty (sFlt-1/PIGF Ratio $+2 S D(R))$ and to the lower bound (LB) of the ratio uncertainty (sFlt-1/PIGF Ratio - $2 S D(R)$ ) were UB $=1.15$ ratio -0.66 and $\mathrm{LB}=0.84$ ratio +0.66 , respectively (Figure 1 ). For the Roche assay, the ratio the equations corresponding to the UB of the ratio uncertainty and to the LB of the ratio uncertainty were $U B=1.11$ ratio +0.004 and $L B=0.89$ ratio -0.004 , respectively. For both the Roche and Kryptor assays, the ratio uncertainty $( \pm 2 S D(R)$ ) interval bounds were linearly proportional to the sFIt-1/PIGF ratio value $\left(r^{2}>0.99\right)$ both for the upper and lower bounds. Based on these results, uncertainties can be calculated for any proposed cut-off values. For example, a ratio value of 38 encompasses a range of 34 to 42 for Elecsys. Likewise, a ratio value of 110 encompasses a range of 100 to 123 and 96 to 129 for the Elecsys and Kryptor assays, respectively. When applied to cut-off values, uncertainty allows areas of uncertainty to be defined. When ratio value uncertainty crosses a cut-off value, the ratio value is considered within the area of uncertainty. It should be noted that for all clinically relevant ratios, the ranges vary by manufacturer and are wider with Kryptor assays above a ratio value of 7 . The areas of uncertainty calculated for each proposed sFIt-1 and PIGF cut-off are summarized in Tables 2 and 3. 


\section{Discussion:}

All staff involved in patient care must be aware that uncertainty is associated with each result. More specifically, standard ISO 15189 states that each biological result should be associated with its uncertainty in order to help clinicians interpret results [10]. This crucial issue has recently been highlighted by Braga et al. and by Plebani et al. [11-12]. This is especially important when cut-off values have potential clinical implications, as in the case of the sFIt-1/PIGF ratio. Results within the range of the cut-off values must be interpreted with caution by the physician. It is therefore necessary to establish whether a result significantly crosses a cut-off value; this depends on the assay used by the laboratory. One way to achieve this goal is to establish and provide trustworthy uncertainties associated with results and the uncertainty derived from the area of uncertainty around the cut-off values to indicate if a result should be interpreted carefully [6]. Due to precision differences between the PIGF and sFlt-1 assays, the same ratio value obtained with different immunoassays can demonstrate different uncertainties depending on the analytical characteristics of the assay used. Changes in the sFIt-1/PIGF ratio of the denominator and the numerator can have a huge effect on the final ratio result. In the case of the sFIt-1/PIGF ratio, previous studies have pointed out that instead of sFIt-1, PIGF results differ among various immunoassays and demonstrate a bias depending on the concentration being tested $[8,13]$. Here, this issue was addressed by using two different ratio uncertainty calculations [9]. IQC was used to calculate a confidence interval $(95 \% \mathrm{Cl})$ for all ratio results, as well as the uncertainty area associated with cut-off values, to adjust the interpretation based on a single cut-off. When a ratio value is located in an area of uncertainty, the probability that the true ratio result is above or below the cut-off value should not be dismissed. Conversely, ratio values outside the area of uncertainty exclude cut-off values with a $95 \%$ probability. This approach can be useful when interpreting diagnosis, prognosis and decision-making in the context of PE. Rather than use external quality controls, which are conventionally used to estimate uncertainty and bias, the decision was made to use IQC because of larger amount of available IQC data.

Roche assays performed with Elecsys immunoassays on Cobas platforms revolutionized the measurement of sFIt-1 and PIGF and offered different cut-off values for assessing the prognosis and/or diagnosis of $\mathrm{PE}[5,14,15]$. Their interpretation relies on the gestational age associated with the ratio value. The cut-off values for short-term prediction of PE was based on the conclusions of the PROGNOSIS study, which found a strong negative predictive value for PE onset for a ratio < 38 [5]. Verlohren et al. showed that with Roche immunoassays a ratio $<33$ could possibly rule out the diagnosis of $P E$, whereas a ratio $\geq 85$ and a ratio $\geq 110$ between 20 and 34 weeks of gestation (WG) and beyond $34 \mathrm{WG}$, respectively, confirmed a diagnosis of $\mathrm{PE}[5,14,15,16]$. To date, the ratio cut-off values in these guidelines have been derived from Roche assays $[17,15]$. In 2012 , the prognosis value 
within 48 hours for high risk adverse events was determined with Roche and corresponded to a ratio $>201$ for late-onset PE and to a ratio > 655 ( $\geq 34 \mathrm{GW}$ ) for early-onset PE (<34 GW), respectively [18]. A similar cut-off value of 655 had been confirmed using the Roche assay in early-onset PE in 2014 [19]. In 2015, PIGF and sFlt-1 automated assays were developed with the Kryptor analyzer (Thermo Fisher) and were compared to Elecsys (Roche) results [20]. Both the analytical characteristics and diagnostic performance for $\mathrm{PE}$, judged by comparing ROC curves between the two assays, were similar, but the optimal ratio cut-off values for detecting PE were different (99.2 for Kryptor and 70.3 for Roche). Conversely, the diagnostic Thermo Fisher cut-off values were not determined. Overall, van Helden et al. found that the Thermo Fisher assay had a higher sensitivity and specificity for the diagnosis of earlyonset PE but a higher sensitivity and a lower specificity for the diagnosis of late-onset PE [20]. Nevertheless, the authors concluded that the Roche assay established cut-off values that could be used with both manufacturers. In 2015, Andersen et al. also compared the Roche and Thermo Fisher assays. They found that the diagnostic performance was comparable but failed to define specific cut-off for Kryptor angiogenic factors [21]. In a recent study, Stepan et al. compared the Roche Elecsys and Kryptor assays in a cohort of pregnant women with and without PE. They found a marked difference between the ratio's values with both analyzers, especially in the low range of sFlt-1/PIGF. This was due to lower Kryptor PIGF values compared to the Roche PIGF assay [8]. Notably, the PIGF values were lowered by at least $50 \%$ using Kryptor assays when compared to Roche assays. Consequently, Kryptor sFIt1/PIGF ratios were $90 \%$ higher than Elecsys ratios with the increase reaching $148 \%$ within the newonset PE group. The authors concluded that it was impossible to apply the same cut-off values defined by Roche to Thermo Fisher results. A case report illustrates the difficulty of interchangeability between these immunoassays [13]. Based on the relationship observed between PIGF assays, Cheng et al. recently proposed an equation between the log-transformed sFIt1/PIGF ratio of the two assays [22]. It proposed transforming the value of Roche PIGF results into Kryptor assay results as follows:

Kryptor ratio $\left.=e^{1.1488 * \log (\text { Roche ratio })-0.1637}\right)$. Consequently, the sFlt1/PIGF ratio results of $33,38,85$ and 110 obtained with the Roche assay could be "transformed" into ratio results of 47, 55, 140 and 188 , respectively, with the Kryptor assay. Conversely, Simón et al. performed a case-control study with 21 cases and compared the performance of the NICE guideline cut-offs between Roche and Kryptor for short-term prediction of PE [23]. Although they confirmed that Kryptor PIGF values were lower and proposed their own equation to transform Kryptor ratio results into Roche results, they considered that Roche cut-offs were valid with the Kryptor assay. Nevertheless, Simón et al. concur that their equation must be used with caution since it has not been clinically validated by other studies and, in accordance with Lefevre et al., concluded that Kryptor cut-offs should be established with a clinical study. 
It seems to us that pending clinical studies aiming at establishing Kryptor sFlt-1/PIGF ratio cut-offs, caution is advised for low ratio value. Thus, Roche cut-offs, which are more stringent, should be used for short-term prediction of PE (38) and PE rule-out (33).

A clinical study aimed at establishing the diagnostic accuracy of Kryptor sFlt-1/PIGF was performed [24]. Based on a cohort of 169 uneventful pregnancies and 46 pregnancies with PE-related outcomes Dröge et al. found an optimal cut-off of 103 for diagnosing PE and PE-related adverse outcomes, with a sensitivity of $88 \%$ and a specificity of $92 \%$ consistent with the 99.2 optimal cut-off found by van Helden et al. for diagnosing PE [20]. It should be noted that sFIt-1/PIGF AOU corresponding to 103 and 99.2 are 90 to 121 and 86 to 106, respectively, thus encompassing the same ratio range.

Since uncertainty is much lower than the bias observed between the PIGF results [20, 21], adding uncertainty to the crude ratio results would only slightly modify the interpretation of the sFlt1/PIGF ratio. Nevertheless, Kryptor cut-offs of 103 or 99 combined with uncertainty could significantly improve the clinical interpretation of the sFIt-1/PIGF ratio in the context of PE [5]. For example, in a case report, pregnant women (33 WG) showed a sFIt-1/PIGF ratio of 153 with the Kryptor assay and 83 with the Roche assay [14]. The Roche ratio value of 83 (corresponding to a uncertainty of 74 to 92) is within the area of uncertainty of 85 (77 to 95), meaning that a high risk of PE could not have been excluded for the patients. Interpreted with the Kryptor assay's 103 or 99 cut-off $[24,20]$, the ratio result (153) would still have classified the patients with a high risk of PE with confidence (area of uncertainty 103 or 99.2 and a cut-off of 90 to 121 and 86 to 106, respectively). Uncertainty calculations also provide insight into each assay's performance. We noted that the ratio uncertainty is greater with the Kryptor assays, indicating slightly lower ratio precision. It should be noted that all $\pm 2 S D(R)$ Kryptor assays encompass the Roche $\pm 2 \mathrm{SD}(\mathrm{R})$ ranges when the ratio is above 7 . Consequently, the uncertainty of both assays is within the same area (Figure 1). As expected, when the sFIt1/PIGF ratio increases, its confidence interval increases proportionally. Unlike with Thermo Fisher IQC, Roche IQC is not available within the full range of linearity of the PIGF and sFIt-1 tests. For example, Roche IQC values provide no ratio value above 11.3. More IQCs with different sFlt-1 and PIGF values from both manufacturers would be an improvement and PIGF and sFIt-1 IQC corresponding to high sFIt-1/PIGF ratio values should be encouraged by IQC manufacturers.

\section{Conclusion:}

The uncertainty of each assay can advantageously be updated and refined periodically using future IQCs defined by each laboratory. Since sFIt1/PIGF ratio has been proposed to aid in the diagnosis by exclusion and/or prognosis of preeclampsia and its short-term prediction, the uncertainty of the sFlt1/PIGF ratio can be assessed and laboratories could be able to provide guidance on interpreting sFlt- 
1/PIGF results. Each laboratory therefore should define its own PIGF/sFlt-1 ratio uncertainty, depending on the assays they use and propose their own area of uncertainty in order to increase the quality of interpretation of results.

References:

[1] Redman CW, Sargent IL. Latest advances in understanding preeclampsia. Science 2005;308:15924.

[2] American College of Obstetricians and Gynecologists; Task Force on Hypertension in Pregnancy. Obstet Gynecol 2013; 122; 1122-31.

[3] Powe CE, Levine RJ, Karumanchi SA. Preeclampsia, a disease of the maternal endothelium: the role of antiangiogenic factors and implications for later cardiovascular disease. Circulation 2011;123:285669.

[4] Maynard SE, Min JY, Merchan J, Lim KH, Li J, Mondal S, Libermann TA, Morgan JP et al. Excess placental soluble fms-like tyrosine kinase 1 (sFlt1) may contribute to endothelial dysfunction, hypertension, and proteinuria in preeclampsia. J Clin Invest 2003; 111:649-58.

[5] Zeisler H, Llurba E, Chantraine F, Vatish M, Staff AC, Sennström M, Olovsson M, et al. Predictive value of the sFlt-1:PIGF ratio in women with suspected preeclampsia. N Engl J Med 2016 ;374:13-22.

[6] Ceriotti F. Deriving proper measurement uncertainty from Internal Quality Control data: An impossible mission? Clin Biochem 2018;57:37-40.

[7] Coskun A, Oosterhuis WP. Statistical distributions commonly used in measurement uncertainty in laboratory medicine. Biochem Med (Zagreb) 2020 Feb 15;30(1):010101. doi: 10.11613/BM.2020.010101.

[8] Stepan H, Hund M, Dilba P, Sillman J, Schlembach D. Elecsys ${ }^{\circledR}$ and Kryptor immunoassays for the measurement of sFlt-1 and PIGF to aid preeclampsia diagnosis: are they comparable? Clin Chem Lab Med 2019;57:1339-1348.

[9] Shackleford DM, Jamsen KM. Quantifying uncertainty in the ratio of two measured variables: a recap and example. J Pharm Sci 2016; 105:3462-3463.

[10] ISO 15189:2012 Medical laboratories: requirements for quality and competence. 
[11] Braga F, Panteghini M. The utility of measurement uncertainty in medical laboratories. Clin Chem Lab Med 2020 Mar 3. Epub ahead of print. pii: /j/cclm.ahead-of-print/cclm-2019-1336/cclm-20191336.xml. doi: 10.1515/cclm-2019-1336.

[12] Plebani M, Padoan A, Sciacovelli L. Measurement uncertainty: light in the shadows. Clin Chem Lab Med 2020 Mar 5. Epub ahead of print pii: /j/cclm.ahead-of-print/cclm-2020-0134/cclm-20200134.xml. doi: 10.1515/cclm-2020-0134.

[13] Lefèvre G, Hertig A, Guibourdenche J, Lévy P, Bailleul S, Drouin D, et al. Decision-making based on sFIt-1/PIGF ratios: are immunoassay results interchangeable for diagnosis or prognosis of preeclampsia? Clin Chem Lab Med 2020 Apr 1. Epub ahead of print pii: /j/cclm.ahead-of-print/cclm2020-0084/cclm-2020-0084.xml. doi: 10.1515/cclm-2020-0084.

[14] Verlohren S, Galindo A, Schlembach D, Zeisler H, Herraiz I, Moertl MG, P et al. An automated method for the determination of the sFlt-1/PIGF ratio in the assessment of preeclampsia. Am J Obstet Gynecol 2010;202:161.e1-161.e11.

[15] Verlohren S, Herraiz I, Lapaire O, Schlembach D, Zeisler H, Calda P, et al. New gestational phasespecific cutoff values for the use of the soluble fms-like tyrosine kinase-1/placental growth factor ratio as a diagnostic test for preeclampsia. Hypertension 2014;63:346-52.

[16] Stepan H, Herraiz I, Schlembach D, Verlohren S, Brennecke S, Chantraine F et al. Implementation of the sFIt-1/PIGF ratio for prediction and diagnosis of pre-eclampsia in singleton pregnancy: implications for clinical practice. Ultrasound Obstet Gynecol 2015;45:241-6.

[17] NICE guidelines: National Institute for Health and Care Excellence (NICE). PIGF-based testing to help diagnose suspected pre-eclampsia (Triage PIGF test, Elecsys immunoassay sFIt-1/PIGF ratio, DELFIA Xpress PIGF 1-2-3 test, and BRAHMS sFlt-1 Kryptor/BRAHMS PIGF plus Kryptor PE ratio). London: NICE; 2016.

[18] Verlohren S, Herraiz I, Lapaire O, Schlembach D, Moertl M, Zeisler H, Calda P et al. The sFIt-1/PIGF ratio in different types of hypertensive pregnancy disorders and its prognostic potential in preeclamptic patients. Am J Obstet Gynecol 2012;206:58.e1-8.

[19] Gómez-Arriaga PI, Herraiz I, López-Jiménez EA, Escribano D, Denk B, Galindo A. Uterine artery Doppler and sFIt-1/PIGF ratio: prognostic value in early-onset pre-eclampsia. Ultrasound Obstet Gynecol 2014;43:525-32. 
[20] van Helden J, Weiskirchen R. Analytical evaluation of the novel soluble fms-like tyrosine kinase 1 and placental growth factor assays for the diagnosis of preeclampsia. Clin Biochem 2015;48:1113-9.

[21] Andersen LB, Frederiksen-Møller B, Work Havelund K, Dechend R, Jørgensen JS, Jensen BL, et al. Diagnosis of preeclampsia with soluble Fms-like tyrosine kinase 1/placental growth factor ratio: an inter-assay comparison. J Am Soc Hypertens 2015;9:86-96.

[22] Cheng YKY, Poon LCY, Shennan A, Leung TY, Sahota DS. Inter-manufacturer comparison of automated immunoassays for the measurement of soluble FMS-like tyrosine kinase- 1 and placental growth factor. Pregnancy Hypertens 2019;17:165-171.

[23] Simón E, Herraiz I, Villalaín C, Gómez-Arriaga PI, Quezada MS, López-Jiménez EA, Galindo A. Correlation of Kryptor and Elecsys ${ }^{\circledast}$ immunoassay sFlt-1/PIGF ratio on early diagnosis of preeclampsia and fetal growth restriction: A case-control study. Pregnancy Hypertens 2020 3;20:44-49.

[24] Dröge LA, Höller A, Ehrlich L, Verlohren S, Henrich W, Perschel FH. Diagnosis of preeclampsia and fetal growth restriction with the sFlt-1/PIGF ratio: Diagnostic accuracy of the automated immunoassay Kryptor $^{\circledast}$. Pregnancy Hypertens 2017;8:31-36

[25] Stepan H, Kuse-Föhl S, Klockenbusch W, Rath W, Schauf B, Walther T, Schlembach D. Diagnosis and treatment of hypertensive pregnancy disorders. Guideline of DGGG (S1-Level, AWMF Registry No. 015/018, December 2013) Geburtshilfe Frauenheilkd 2015; 75: 900-914. 
Table 1: sFIt-1/PIGF IQC results for Roche Elecsys and Thermo Fisher Kryptor

$\mathrm{n}_{1}=$ total number of results; $\mathrm{n}_{2}=$ number of IQCS analyzed per batch; $\mathrm{N}=$ number of batches.

\begin{tabular}{cccccc}
\hline Assay & IQC level & $\mathrm{n}_{1}$ & $\mathrm{n}_{2}$ & $\mathrm{~N}$ & $\begin{array}{c}\text { Approximate } \\
\text { concentration } \\
\text { (pg/mL) }\end{array}$ \\
\hline Kryptor PIGF & Low & 706 & 56 to 246 & 5 & 32 \\
\cline { 2 - 6 } & Medium & 706 & 58 to 243 & 5 & 106 \\
\cline { 2 - 6 } & High & 705 & 59 to 241 & 5 & 425 \\
\hline Kryptor sFlt-1 & Low & 661 & 29 to 317 & 5 & 1455 \\
\cline { 2 - 6 } & Medium & 662 & 29 to 316 & 5 & 2957 \\
\cline { 2 - 6 } & High & 660 & 27 to 317 & 5 & 9840 \\
\hline $\begin{array}{c}\text { Roche Elecsys } \\
\text { PIGF }\end{array}$ & Low & 1050 & 121 to 354 & 4 & 983 \\
\cline { 2 - 6 } & High & 1049 & 102 to 362 & 4 & 982 \\
\hline Roche Elecsys & Low & 1034 & 115 to 336 & 4 & \\
\cline { 2 - 6 } sFlt-1 & High & 1033 & 100 to 356 & 4 & 104 \\
\hline
\end{tabular}

IQC: internal quality controls 
Table 2: sFIt-1/PIGF ratio area of uncertainty (AOU) calculated for Roche Elecsys diagnosis and prognosis cut-off values according to NICE [17] and Swiss, Austrian and German guidelines [25].

\begin{tabular}{cccc}
\hline & & sFlt-1/PIGF cut-offs & sFlt-1/PIGF AOU \\
\hline Purpose & WG & & \\
\hline $\begin{array}{c}\text { Aid in PE diagnosis } \\
{[17]}\end{array}$ & 20 to $33+6$ days & rule-out: $<33$ & 30 to 37 \\
\cline { 2 - 4 } & & rule-in: $>85$ & 30 to 95 \\
\cline { 2 - 4 } & $>34$ WG & rule-out: $<33$ & 100 to 123 \\
\hline $\begin{array}{c}\text { Short-term prediction } \\
\text { of PE } \\
{[17]}\end{array}$ & $24<$ WG $<36$ & rule-in: $>110$ & 34 to 42 \\
\cline { 2 - 4 } & & rule-out*: $<38$ & 34 to 42 \\
\hline $\begin{array}{c}\text { Hospitalization for } \\
\text { PE support [25] }\end{array}$ & Not defined & rule-in**: $>38$ & 77 to 95 \\
\hline
\end{tabular}

*1-week rule-out for pre-eclampsia.

** 4-week rule-in for pre-eclampsia.

AOU: area of uncertainty 
Table 3: sFIt-1/PIGF ratio area of uncertainty (AOU) calculated for Thermo Fisher cut-off values (proposed by Dröge et al. [24] and Van Helden et al. [20]).

\begin{tabular}{|c|c|c|}
\hline & Proposed sFlt-1/PIGF Kryptor cut-off & sFlt-1/PIGF AOU \\
\hline \multicolumn{3}{|l|}{ Purpose } \\
\hline \multirow{2}{*}{$\begin{array}{c}\text { Aid in PE diagnosis and PE } \\
\text { adverse-related outcome [24] }\end{array}$} & rule-out: $<103$ & 90 to 121 \\
\hline & rule-in: > 103 & 90 to 121 \\
\hline \multirow[t]{2}{*}{ Aid in PE diagnosis [20] } & rule-out: $<99.2$ & 86 to 106 \\
\hline & rule-in: $>99.2$ & 86 to 106 \\
\hline
\end{tabular}


Figure 1: Uncertainty of the sFIt-1/PIGF ratio: lower and upper bound of $\pm 2 \mathrm{SD}(\mathrm{R})$ range as a function of the sFlt-1/PIGF ratio results (Roche Elecsys and Thermo Fisher Kryptor assays); results in $\log _{10}$ scale.

Thermo Fisher Kryptor lower bound

Thermo Fisher Kryptor upper bound

Roche Elecsys lower bound

$\times \quad$ Roche Elecsys upper bound

lower and upper bound $\pm 2 \mathrm{SD}(\mathrm{R})$ for Roche Elecsys

lower and upper bound $\pm 2 \mathrm{SD}(\mathrm{R})$ for Thermo Fisher Kryptor

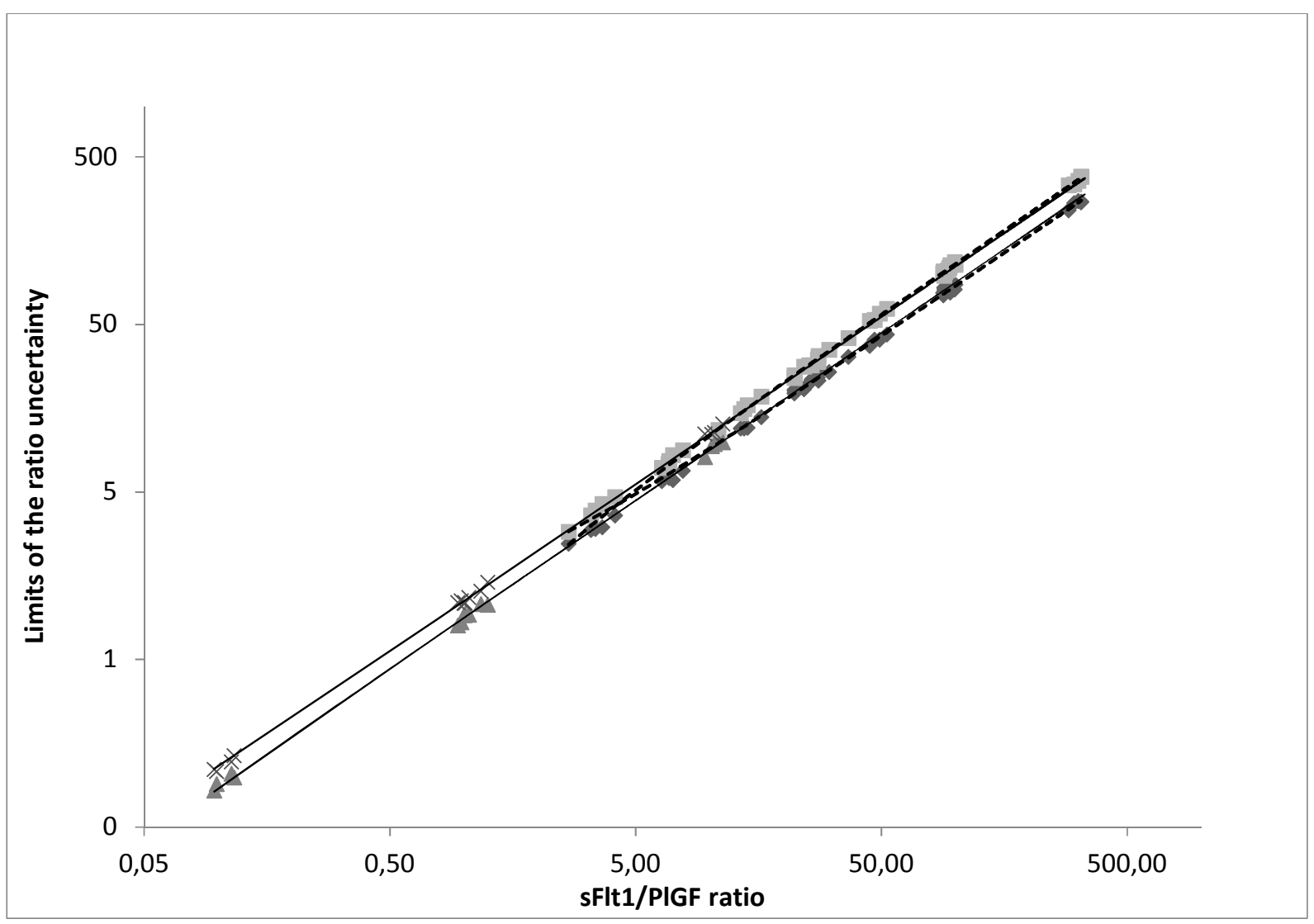

